\title{
Patient-Derived-Tumor-Xenograft: modeling cancer for basic and translational cancer research
}

\author{
Danilo Fiore $^{1}$, Filomena Di Giacomo ${ }^{1}$, Peter W. Kyriakides ${ }^{1}$ and Giorgio Inghirami ${ }^{1,2,3}$ \\ ${ }^{1}$ Department of Pathology and Laboratory Medicine, Weill Cornell Medicine, New York, NY, USA \\ ${ }^{2}$ Department of Molecular Biotechnology and Health Science and Center for Experimental Research and Medical Studies (CeRMS), University of Torino, Torino, \\ 10126 Italy \\ ${ }^{3}$ Department of Pathology, and NYU Cancer Center, New York University School of Medicine, New York, NY, 10016 USA
}

\begin{abstract}
Patient-Derived-Tumor-Xenografts (PDTX) represent one of the most promising platforms to model human cancer and its complexity. PDTX are able to closely recapitulate the principal features of donor tumors, and remain fairly stable along the passages, rendering them an ideal tool to serve as powerful and predictive models in oncology. Here we aimed to overview our current understanding of PDTX, and their potential applications in basic and translational cancer research. We briefly describe the methodological aspects of PDTX generation, and then focus on the usefulness of PDTX in tumor heterogeneity and clonal evolution studies, as well as their usage to dissect the host microenvironment and the emergence of drug resistance. We also focus on the key role of PDTX in the discovery of biomarkers and drug screening development. The limitations and future perspectives to further improve the PDTX models are also discussed.
\end{abstract}

\section{Introduction}

In the last few decades, massive efforts have been devoted to understanding the pathobiology of human cancers. Despite remarkable advances, elucidating the mechanisms underlying tumor establishment and progression still appears incomplete [1]. This lack of deep understanding is responsible for the partial success in the cure of cancer patients and our failure to eradicate cancers in the vast majority of patients [2-4]. Among many of the caveats, which have weakened major successes, a major issue remains in the lack of reliable models that can extensively reproduce human cancers and all their multifaceted features $[3,5]$. Therefore, the development of novel models that can more accurately reproduce tumor heterogeneity and predict in vivo drug sensitivity and response to patients is necessary to effectively move forward in cancer research and clinical practice.

Cancer cell lines still represent the most common models in cancer research and anticancer drug discovery. These simplified models, such as $2 \mathrm{D}$ cultures, have objective advantages, however they lack appropriate cell-cell and cell-matrix interactions, and the prerequisites required to fully maintain the appropriate oncogenic phenotype. Moreover, all of them have undergone enormous adjustments and are the end product of strict clonal selection, which do not fully represent human cancers and their heterogeneous nature. These events frequently de-route the cancer prone signaling pathways operating in tumor cells within their natural habitat. To overcome these limitations, many attempts have been made to culture purified populations of primary cells either in $2 \mathrm{D}$ or as tumor fragments/organoids. Nevertheless, primary cells can also rapidly lose their features, or alternately can only survive for relatively short period of times, further underscoring the intricate requirements of cancer cells [6,7].

Of note, organoid cultures provide more effective in vitro cancer models, which more closely mimic the tumor growth of primary human cancers [8]. They are sustained by different physical supports that aim to mimic the tumor microenvironment. Interestingly, the extra-cellular matrix of organoids can be functionalized to provide more appropriate stimuli $[9,10]$. Because clinical tumor responses to therapeutic agents can be highly unpredictable, organoids represent a powerful tool for both drug screening and basic cancer research $[11,12]$. However, organoid models are often comprised of individual or at best just a few tumoral elements. Indeed, models with multiple cell types, the appropriate organization and the appropriate cell-cell or cell-matrix interactions remain hardly achievable. Though they are promising biological tools representing a good balance between feasibility and stability. However we do not have yet developed fully highly comprehensive tools, which may overcome the limitations of either $2 \mathrm{D}$ or current $3 \mathrm{D}$ culture systems $[8,11,12]$.

The field of Patient-Derived-Tumor-Xenografts (PDTX), which was first described more than 40 years ago, may represent a valuable option for cancer research and drug discovery [13,14]. Since then, the availability of unique immune-deficient mice has significantly increased [15]. This has led to improved tumor engraftment rates and a wider usage of PDTX models. Interestingly, the histopathology of PDTX tumors closely mimics those of the donor lesions. A plethora of evidence, including high fidelity in mutational status, transcriptome, histology, polymorphism and copy number variation, also supports the notion that PDTX models remarkably resemble the pathophysiology of human tumors more closely than traditional Cancer-derived xenograft (CDX) models [16]. This convincing data suggests PDTX

Correspondence to: Danilo Fiore, Department of Pathology and Laboratory Medicine, Weill Cornell Medicine, New York, NY, USA. E-mail: daf2038@med.cornell.edu

Key words: pdtx, classifier, microenvironment, drug screening

Received: April 12, 2017; Accepted: May 11, 2017; Published: May 14, 2017 
are informative models to study clonal evolution along serial passages, at the rates reported in primary tumors [17-24]. Notably, PDTX grow within a rich host environment and the relationship between human tumors and mouse host elements have been proven to be critical for the successful engraftment, growth and response to therapy [25]. Therefore PDTX can be used not only to study tumor cells, but also to investigate the role and function of the stroma/host, providing new opportunities to explore and dissect the protumorigenic role of cancer niches. Collectively, several studies have now demonstrated that PDTX represent effective models to test drugs that target cancer elements and modulate tumor vasculature and/or the stromal compartment $[26,27]$.

Unfortunately, PDTX too have sizable limitations. For example, it is still unclear whether the resistance or sensitivity of patient tumors toward cancer chemotherapeutics is retained in PDTX models. This question represents the central focus of the current debate over the utility of PDTX models. Another important barrier is the imperfect cross talk between murine and human cells and the lack of key features of the immune machinery in immune-compromised mice. At this point, the usage of PDTX models to study drugs whose pharmacological activities target the host immune system, including immunotherapeutic compounds, is highly limited [18]. This is a limitation that could be at least in part be overcome by humanized PDTX models [28]. At this point, PDTX represent reliable and effective tools capable of providing new avenues to discover the enormous complexity of cancer cells and their microenvironment. Lastly, although they provide an unprecedented opportunity, the findings emerging from these models need to be integrated and cross-validated with those derived from other systems and accurately annotated by genomics read outs.

Herein, we will focus on the principles of PDTX generation, and discuss how these models may be envisioned. Limitations of representative models will also be discussed and alternatives to overcome them entertained.

\section{PDTX generation and characterization}

The ability to grow human tumor tissues in host recipients was developed more than 40 years ago by Cobb LM [13]. However, only in the last few decades have PDTX been widely used in cancer research. Although customized methodologies have been employed, their general rationale and objectives remain similar [22,29,30]. In the case of solid neoplasms, after sample collection, tumors are rapidly processed and tissue fragments $\left(\approx 3 \mathrm{~mm}^{3}\right)$ are implanted via multiple routes, more frequently subcutaneously or orthotopically. Contrary to previous approaches, which took advantage of single cell suspensions generated after mechanical or enzymatic manipulation, the implantation of tissue fragments provides a rational advantage in maintaining the overall organization of the neoplastic microenvironement and ultimately preserves neoplastic niches. In the setting of liquid tumors, cancer cells are instead purified (gradient separation etc.) and implanted (intravenous, intraperitoneal, intraspleen-liver, intrabone) in immunocompromised host animals. Orthotopical implantation and/or injection represent additional options, to allow tumor expansion in the same organ of the parental tumor (mammary fat pad, brain, ovary), and consequently it is anticipated that these derived PDTX may represent more predictive models. The time of engraftment varies considerably between different cancers and also among different xenograft belonging to the same tumor type. In general, the growth takes about 2-3 months, but longer periods of time ( $>6-9$ month) are not unusual. Of note, in many models there is an increasing fitness along passages with a shorting of the time needed to develop tumors. This suggests the selection of best-fitted populations can occur along propagation, demonstrating that these models are highly dynamic. Overall, this provides valuable powerful tools to explore cancer evolution and progression, as demonstrated in a study of Ding et al who reported that the mutations acquired by a breast PDTX across different generations could be found in the metastatic sites [31]. A similar scenario has been described in leukemia PDTX models [32].

Technically, different factors can influence the rate of engraftment, such as the quality of the patient derived material, tumor type, stage of differentiation, drug resistance, time of implant, number of cells, and routes of implantation. Perhaps the most critical variable is the selection of mouse strain. The development of RAG or NOD/SCID/ IL2R $\gamma^{\text {null }}$ (NSG) mice represents a major achievement and has improved engraftment of primary human tumor specimens [33]. NSG mice are Scid deficient (bearing a DNA repair complex protein Prkdc mutation) resulting in profound defects in both B and T cells. They also harbor a target mutation of the gene encoding the IL2-receptor common $y$ chain (IL2 rg $^{\text {null }}$ ) and the lack of signaling through IL2rg results in the functional impairment of NK cells, thus severely compromising both the innate and adaptive immunities in these animals [15]. Moreover, engineered NSG mice have emerged for specific application (i.e. AML) and to generated more reliable humanized mice.

Testing the reliability of PDTX as a photocopy of the primary tumor remains a necessity for the appropriate usage and interpretation of the model data. This can be approached using different and integrated readouts. Routine histology, immunohistochemical analyses can probe many of the PDTX phenotyping features (tissue structure, etc), while genomics (target sequencing, Exome-Seq and RNA-Seq) can reveal the degree of similarities among primary and derived xenografts (mutations, copy number variations, SNPs). Both platforms can provide data on the contribution of normal human and mouse cells and the balance between these elements. This is a critical issue, considering that in general human host cells are lost rapidly in the first passages and are substituted by the intratumoral infiltration of normal mouse elements; a phenomenon that is highly dynamic along passages [25]. Moreover, biological features such as drug sensitivity and metastatic spreading need to be tested as well and ideally they should be maintained through serial transfers in vivo.

\section{PDTX in basic cancer research}

\section{Modeling tumor heterogeneity and clonal evolution}

During tumor initiation and progression, cancer cells acquire mutations/defects, which increase their oncogenic potential, ultimately leading to unchecked growth and host evasion [34]. Along this process, a multitude of individual clones may emerge, whose destiny largely relies of the equilibrium between the cancer and compartments, which are mutually influenced and continuously reshaped $[23,35,36]$. The highly heterogeneous and dynamic nature of cancers is further complicated by inter-tumor as well as intra-tumor heterogeneity [37,38]. The latter is driven by both cellular (genomic and epigenomic heterogeneity) and stromal interactions [39]. Clones evolve dynamically in space and time following principles of Darwinian evolution, generating remarkable features, such as drug resistance and metastasis. The existence of intratumoral subclone diversity elucidates the enormous flexibility of cancer cells in response to different stresses, including chemotherapy $[18,40,41]$. This heterogeneity is largely lost when tumor cells are propagated in vitro, a scenario that facilitates the emergence of few or a single dominant clone that most efficiently fits to the new in vitro conditions. This is epitomized by a recent study in which Daniel et 
al. have shown marked changes of critical protumorigenic pathways (chemosensitivity, stromal interactions, and targeted therapies) in cultured lines of small cell lung cancers compared to their parental PDTX. Interestingly, these changes could not be reverted once the in vitro propagated cells were re-implanted into mice. Instead, PDTX retain the original tumor heterogeneity, thus allowing for clonal dynamic studies [6,25,30,42-46]. Moreover, Eirew et al. used deep genome and single cell sequencing methods to demonstrate an ongoing clonal selection in both primary and metastatic breast tumors, with the expansion of clones sharing recurrent patterns. However, in cases undergoing a limited selection within the first passages, a subsequent evolution did not occur. The authors went further to show that clonal expansion patterns were reproducibly seen in independent grafts, implying that the selection is a non-random process. This process depends, however, on the mutation genotype (or epigenotype) that defines each individual cancer as well as the mutual relationship with the environment [47]. On the same line, Ebinger S. et al. have isolated a subclone from PDTX derived from rare relapse-inducing cells of acute lymphoblastic leukemia (ALL) that show dormancy, therapy resistance and stem-like properties. By using single-cell RNASeq data, the authors revealed substantial similarity with primary ALL cells isolated from pediatric and adult patients at minimal residual disease (MRD) [32]. Using this later approach, Kim et al. were able to demonstrate a candidate tumor cell subgroup associated with anti-cancer drug resistance in viable lung adenocarcinoma in PDTX [48]. Additionally, Nguyen LV et al. have applied a DNA barcoding technology to track the clonal evolution of breast PDTX. They demonstrated a continuing diversity in the growth activities of the individual clones that contribute to the combined exponential growth of tumors. Interestingly, the extremely instable clonal landscape supports the hypothesis that clonal expansion may be gained, lost or transiently arrested due to different stimuli or stresses, providing an attractive explanation of the dormant clones in vivo [49]. In conclusion, PDTX appear to be highly effective models to dissect tumor heterogeneity, and this will eventually provide critical data to a better understand the mechanisms underlying tumor biology, drug resistance, and propagation of human cancers.

\section{PDTX as a tool to explore microenvironment}

In the last decade, tumor microenvironment emerged as a key player in tumorigenesis, and it now is widely considered a major hallmark of cancers [50]. The tumor stroma comprises numerous cell types i.e. endothelial cells, cancer-associated fibroblasts (CAFs), mesenchymal stem cells, and immune cells (such as lymphocytes and tumorassociated macrophages). Together with tumor cells, stromal elements functionalize the malignant niche responsible for cancer growth, survival and the development of resistance/relapsing phenotypes [50]. Understanding the mechanisms regulating host-cancer relationships will be essential to design and test more effective therapeutic strategies. Indeed, it is plausible that the poor clinical success of several therapies may be largely due to the erroneous models (cell lines and cell line xenografts) used in conventional drug discovery pipelines that do not adequately account for the microenvironment.

The functional contribution of the stroma in PDTX is still quite controversial. Tumor fragments used to establish PDTX models contain tumor and stromal cells, as well as the extracellular matrix. Human stroma is detectable in early passages, but it is completely replaced along transplantations by mouse stroma [25,51]. In fact, recent studies clearly demonstrated how murine cells functionally replaced human stroma to recreate malignant niches closely mimicking the original human tumor microenvironment. For example, Breakeveldt
$\mathrm{N}$ et al. using neuroblastoma PDTX have reported that mice stroma recapitulate the clinical hallmarks of original primary neuroblastomas, such as the rich vascularization, macrophage infiltration, and CAF and ECM composition. Moreover, the authors observed that patientderived endothelial cells could form blood vessels, although tumor stroma was predominantly replaced with murine stroma [52]. On the same line, Sansone $\mathrm{P}$ et al. have generated PDTX models of luminal breast cancer and isolated cancer-associated fibroblast from hormonal therapy resistant bone metastases. Through their analysis, they found a new process of CAF-microvescicles mediated hormonal therapy resistance [53]. By using next generation sequencing, it is now possible to gain insights into the protumorigenic signals provided by the host [54-56]. In fact, the bioinformatic deconvolution of mouse host reads and human cancers allow for the generation of molecular signatures, which are highly informative of the mechanisms sustained by the cancer environment [57]. Collectively, this new knowledge provides a deeper and more comprehensive understanding of the stroma's role in tumor progression, metastasis and the generation of therapy-related resistant phenotypes.

\section{Short term culture of Patient-derived tumor cells (PDTC): Identification of tumor progression and drugs resistance mechanisms}

The inability of cancer cell lines to faithfully recapitulate inter and intra tumor heterogeneity and their adaption to propagation in vitro has strongly impaired our capability to study human cancers. PDTX represents a promising clinical model to recapitulate the tumor complexity. However, the extensive use of PDTX for multiple and high-throughput studies may prove to be unfeasible considering the enormous resources and costs required. Therefore, the optimization of ex-vivo short-term culture conditions from primary PDTX represents an invaluable source for insight into tumor mechanisms and to perform informative high-throughput drug screening. Recently, Bruna A et al. have generated 27 PDTC from different breast PDTX and demonstrated that PDTC retained the same percentage of mouse stromal cells and the molecular features of the original PDTX. They successfully used these models as a predictive pre-clinical drug-screening platform and to test drug combinations [58]. Furthermore, PDTC can be used to perform co-culture studies or to study cancer stem cells as well as the molecular mechanisms of transformation and drug resistance.

\section{PDTX in translational cancer research}

\section{Forestall treatment failure: Classifier identification}

In the last decade, the enhancement and diffusion of highthroughput sequencing technologies has led to the annotation of the genetic alterations and pathways in individual tumors, allowing for the development of therapies based on the genetic makeup of each individual cancer patient. This approach, once applied to patients, has fostered the design and implementation of new molecular targeted therapeutics to specifically shut down/block key drivers sustaining tumor growth and progression. Although important improvements have been achieved in this arena, the efficacy of anticancer treatments is still largely linked to responses of individual patients. At present, no reliable prediction can be forecasted for many cancers. Acquiring a larger cohort of patient data is imperative to improve the reliability of these predictors and take full advantage of PDTX models. Therefore, the discovery of new predictive and prognostic biomarkers should be a priority in cancer research, allowing patients stratification for specific treatment protocols and to predict clinical compliance and quality of 
responses. Clinical specimens directly obtained from cancer patients represent the best source for the identification of new predictors. However, the difficulties involved in obtaining fresh primary samples deeply impair the capacity to individuate new biomarkers. Because PDTX largely retain the genetic and phenotypical features of original tumors, they represent an ideal and versatile tool to facilitate the identification of tumor specific classifiers [59]. Recent evidence reports a high concordance between PDTX responses and human trials, in particular regarding the discovery of biomarkers. Zembutsu et al. conducted one of the first studies aimed to discover biomarkers from PDTX in 2002. The authors used cDNA microarray technology to define the gene expression profiles of 85 PDTX from 9 different tumor types. This approach highlighted genes significantly associated with positive responses for 9 anticancer drugs and 1578 genes were found to correlate with at least one drug sensitivity, and 333 with different combinations of two or more compounds [60]. More recently, Girotti et al. established a large cohort of 91 melanoma PDTX. Using WES, they integrated the data with ctDNA sequencing and PDTX functional studies to describe a novel strategy for the stratification of naïve patients [61]. Alternatively, PDTX can be used to find protein biomarkers related to drug response. Toward this end, Brown et al. have identified in glioblastoma PDTX, and in corresponding primary tumors, a subset of tumors with similar proteomic profiles carrying high levels of pEGFR, whose tumors were effectively targetable by a selective kinase inhibitor [62]. Similarly, Richmond et al. have identified determinants of therapeutics in ph-like-ALL using PDTX. They performed a global gene expression profile of ALL-PDTX, 6 responders and 6 nonresponders, to birinapant (SMAC mimetic). Using this approach, they found that the efficacy of birinapant strongly depends on TNF $\alpha$ (or other inflammatory cytokines) expression, which could be used as predictive biomarker for birinapant response in ALL patients [63]. Since the capacity to positively engraft breast cancers into host mice depends on the aggressiveness of the primary tumor, Moon et al. were able to show a specific prognostic signature linked to TNBC PDTX engraftment predicting patient survival [64]. Lastly, PDTX can be used to find epigenetic biomarkers to predict patient response, as demonstrated by the study of Gupta and colleagues who demonstrated that the MGMT promoter hypermethylation predicts the efficacy of Temozolomide plus a PARP inhibitor (Veliparib) in GBM PDTX [65].

\section{PDTX models for drug discovery}

One of the major issues in new drug development is linked to the low success rate of developing effective new agents. The poor rate of success of many drug discovery programs is likely due to the usage of conventional preclinical models. Hence, the availability of more informative preclinical models with higher predictive value is of major interest. It is anticipated that an improved drug discovery platform will be highly beneficial in the design and successfully implementation of phase II studies. For their intrinsic features, PDTX represent powerful models to overcome some of the limitations of conventional cell lines. Different studies have recently demonstrated that PDTX can faithfully predict and recapitulate responses seen in clinical trials and can be used for drug screening. With this goal in mind, Townsend et al. have established a large cohort of PDTX from a large number of leukemia and lymphoma patients. These models were used to perform phase II-like clinical trials, testing the efficacy of a new MDM2 inhibitor (CGM097) in B-ALL PDTX. CGM097 conferred a prolonged survival in large majority of PDTX (19/20) with WT p53, while it had a minimal effect in p53 mutated B-ALL PDTX [66]. In solid tumor derived PDTX, Gao et al., having generated an extensive repository of $\sim 1,000$ PDTX, performed a large-scale screen demonstrating inter-patient response heterogeneity applying a 'one animal per model per treatment' approach. With this new strategy, they successfully identified novel therapies that cell line models failed to discover. Moreover, they were also able to confirm that the responses of the PDTX closely mimic those seen in patients, disproving the faithfulness of $2 \mathrm{D}$ models (i.e. anti-IGFR1). It plausible that the discrepancies in drug responses between in vitro and in vivo data may be due to interactions and protumorigenic signals of the host [67]. Toward this end, Sugimoto and colleagues have demonstrated that the co-culture of BLS4 fibroblast reticular mouse cell lines strongly sustained PDTX primary cell viability, allowing an effective ex vivo screening with a library of 2613 compounds. Interestingly, using this approach the authors found a metabolic dependency and identify a novel Achilles' heel of lymphoma cells which could be effectively targeted by the pyruvinium pamoate (PP), an FDA-approved classical anthelminthic compound, inhibiting glutathione-mediated pathways [26]. In the recent years a plethora of studies have been clearly shown the effectiveness of these models in many different arenas. Due to space limitations we have discuss few of them but comprehensive discussion of the topic have been reviewed buy several groups [41,68-73].

Collectively, these findings provide a strong rationale for the usage of PDTX models within translational and drug discovery programs. Lastly, having demonstrated that each individual tumor has a unique property/phenotype, the construction of large PDTX repositories should become a mandatory objective for the scientific community. This will be only achieved if integrated agendas and international efforts are established embracing both academic institutions and private industries.

\section{Limitations and challenges}

The increasing knowledge in cancer biology has clearly pointed out the limitation of many animal models and the necessity to take full advantage of emerging technologies (i.e. genome editing, ultra deep sequencing, single cell genomics, integrated omics etc.). Although these approaches have opened new avenues in cancer biology, a dedicated effort needs to place to create truly representative models that fully describe/recapitulate the complexity of human cancer. PDTX may answer some of these questions. Though PDTX represents a new frontier to more comprehensively explore cancer oncology, multiple limitations remain to be solved. Some of these are technical, such as the high costs of experiments, dedicated infrastructures and specialized personnel as well as the availability of primary samples and the frequent long interval required for the emergence of successful PDTX lines. Lastly, poor rates of engraftment remain a serious roadblock for many types of human cancers (for example prostate, tumors bearing unique genotypes, i.e. EGFR positive non small lung cancers). Another relevant pitfall lies with the loss of intratumoral heterogeneity along serial passages. Lastly, the absence of a functional immune system shaping tumor progression and the emergence of spontaneous drug resistance are well known confounding elements. One of the major limitations of PDTX models is the necessity for highly immunocompromised hosts to avoid graft versus host disease. This totally impairs the possibility to use PDTX models for immunotherapeutic studies and approaches, such as vaccines, immune modulators and activators. To overcome this important issue, humanized mouse have recently emerged [74-79]. Different degrees of reconstitution have been applied, including the simple injection of either heterologous or autologous peripheral blood mononuclear cells or alternatively the transplant of CD34-positive human hematopoietic cells. Although very promising, we are still in the early infancy and many obstacles need to be surmounted. The 
generation of class I and II NSG strains and the application of editing technologies should facilitate the generation of effective immune reconstitutions and the creation of strains in which human genes effectively replace their mouse counterparts.

In conclusion, PDTX have emerged as powerful new platforms to effectively probe a plethora of different questions in oncology. Although several issues need to be overcome, these models provide an invaluable opportunity to test and validate novel therapeutic strategies.

\section{Financial support}

This work was partially supported by funds from the Leukemia Lymphoma Society, (SCOR grant) to G.I. and the AIRC 5xMille consortium 'Genetics-driven targeted management of lymphoid malignancies' to G.I.

\section{References}

1. de Bono JS, Ashworth A (2010) Translating cancer research into targeted therapeutics. Nature467: 543-549.[crossref]

2. DiMasi JA, Reichert JM, Feldman L, Malins A (2013) Clinical approval success rates for investigational cancer drugs. Clin Pharmacol Ther 94: 329-335.[crossref]

3. Ellis LM, Fidler IJ (2010) Finding the tumor copycat. Therapy fails, patients don't. Nat Med 16: 974-975.[crossref]

4. Kola I, Landis J (2004) Can the pharmaceutical industry reduce attrition rates? NatRev Drug Discov 3: 711-715.[crossref]

5. Johnson J, Decker S, Zaharevitz D, Rubinstein LV, Venditti JM, et al. (2001) Relationships between drug activity in NCI preclinical in vitro and in vivo models and early clinical trials. Br J Cancer 84: 1424-1431. [crossref]

6. Daniel VC, Marchionni L, Hierman JS, Rhodes JT, Devereux WL, et al (2009) A primary xenograft model of small-cell lung cancer reveals irreversible changes in gene expression imposed by culture in vitro. Cancer Res 69: 3364-3373. [crossref]

7. Gillet JP, Calcagno AM, Varma S, Marino M, Green LJ, et al. (2011) Redefining the relevance of established cancer cell lines to the study of mechanisms of clinical anticancer drug resistance. ProcNatlAcadSci U S A 108: 18708-18713.[crossref]

8. Gao D, Chen Y (2015) Organoid development in cancer genome discovery. CurrOpin Genet Dev30: 42-48.[crossref]

9. Gjorevski N, Sachs N, Manfrin A, Giger S, Bragina ME, et al. (2016) Designer matrices for intestinal stem cell and organoid culture. Nature539: 560-564.[crossref]

10. Murrow LM, Weber RJ, Gartner ZJ (2017) Dissecting the stem cell niche with organoid models: an engineering-based approach. Development 144: 998-1007.[crossref]

11. Yin X, Mead BE, Safaee H, Langer R, Karp JM, et al. (2016) Engineering Stem Cell Organoids. Cell Stem Cell 18: 25-38.[crossref]

12. Young M, Reed KR (2016) Organoids as a Model for Colorectal Cancer. CurrColorectal Cancer Rep 12: 281-287.[crossref]

13. Cobb LM (1973) The behaviour of carcinoma of the large bowel in man following transplantation into immune deprived mice. Br J Cancer 28: 400-411.[crossref]

14. Crystal AS, Shaw AT, Sequist LV, Friboulet L, Niederst MJ, et al. (2014) Patientderived models of acquired resistance can identify effective drug combinations for cancer. Science 346: 1480-1486. [crossref]

15. Racki WJ, Covassin L, Brehm M, Pino S, Ignotz R, et al. (2010) NOD-scid IL2rgamma(null) mouse model of human skin transplantation and allograft rejection. Transplantation 89: 527-536. [crossref]

16. Zhao X, Liu Z, Yu L, Zhang Y, Baxter P, et al. (2012) Global gene expression profiling confirms the molecular fidelity of primary tumor-based orthotopicxenograft mouse models of medulloblastoma. Neuro Oncol 14: 574-583. [crossref]

17. DeRose YS, Wang G, Lin YC, Bernard PS, Buys SS, et al. (2011)Tumor grafts derived from women with breast cancer authentically reflect tumor pathology, growth, metastasis and disease outcomes. Nat Med 17: 1514-1520.[crossref]

18. Hidalgo M, Amant F, Biankin AV, Budinska E, Byrne AT, et al. (2014) Patient-derived xenograft models: an emerging platform for translational cancer research. Cancer Discov 4: 998-1013. [crossref]
19. Loukopoulos P, Kanetaka K, Takamura M, Shibata T, Sakamoto M, et al.(2004) Orthotopic transplantation models of pancreatic adenocarcinoma derived from cell lines and primary tumors and displaying varying metastatic activity. Pancreas 29: 193203. [crossref]

20. Malaney P, Nicosia SV, Davé V (2014) One mouse, one patient paradigm: New avatars of personalized cancer therapy. Cancer Lett344: 1-12.[crossref]

21. Morton CL, Houghton PJ (2007) Establishment of human tumorxenografts in immunodeficient mice. Nat Protoc2: 247-250.[crossref]

22. Tentler JJ, Tan AC, Weekes CD, Jimeno A, Leong S, et al. (2012) Patient-derived tumour xenografts as models for oncology drug development. Nat Rev Clin Oncol 9: 338-350. [crossref]

23. Ding L, Raphael BJ, Chen F, Wend MC (2013) Advances for studying clonal evolution in cancer. Cancer Lett 340: 212-219. [crossref]

24. Massabeau C, Fournier-Bidoz N, Wakil G, Castro Pena P, Viard R, et al. (2012) Implant breast reconstruction followed by radiotherapy: can helical tomotherapy become a standard irradiation treatment? Med Dosim 37: 425-431.

25. Cassidy JW, Caldas C, Bruna A (2015) Maintaining Tumor Heterogeneity in PatientDerived TumorXenografts. Cancer Res 75: 2963-2968.[crossref]

26. Sugimoto K, Hayakawa F, Shimada S, Morishita T, Shimada K, et al. (2015) Discovery of a drug targeting microenvironmental support for lymphoma cells by screening using patient-derived xenograft cells. Sci Rep 5: 13054. [crossref]

27. Ruggeri BA, Camp F, Miknyoczki S (2014) Animal models of disease: pre-clinical animal models of cancer and their applications and utility in drug discovery. BiochemPharmacol87: 150-161.[crossref]

28. Byrne AT, Alferez DG, Amant F, Annibali D, Arribas J, et al. (2017) Interrogating open issues in cancer precision medicine with patient-derived xenografts. Nat Rev Cancer 17: 254-268. [crossref]

29. Calles A, Rubio-Viqueira B, Hidalgo M (2013) Primary human non-small cell lung and pancreatic tumorgraft models--utility and applications in drug discovery and tumor biology. CurrProtocPharmacolChapter 14:Unit 14 26. [crossref]

30. Siolas D, Hannon GJ (2013)Patient-derived tumorxenografts: transforming clinica samples into mouse models. Cancer Res73: 5315-5319. [crossref]

31. Ding L, Ellis MJ, Li S, Larson DE, Chen K, et al. (2010) Genome remodelling in a basal-like breast cancer metastasis and xenograft. Nature 464: 999-1005. [crossref]

32. Ebinger S, Ozdemir EZ, Ziegenhain C, Tiedt S, Castro Alves C, et al. (2016) Characterization of Rare, Dormant, and Therapy-Resistant Cells in Acute Lymphoblastic Leukemia. Cancer Cell30:849-862. [crossref]

33. Ito M, Hiramatsu H, Kobayashi K, Suzue K, Kawahata M, et al. (2002) NOD/SCID/ gamma(c)(null) mouse: an excellent recipient mouse model for engraftment of human cells. Blood 100:3175-3182. [crossref]

34. Pizzi M, Boi M, Bertoni F, Inghirami G (2016) Emerging therapies provide new opportunities to reshape the multifaceted interactions between the immune system and lymphoma cells. Leukemia 30: 1805-1815.

35. Shah SP, Roth A, Goya R, Oloumi A, Ha G, et al. (2012) The clonal and mutationa evolution spectrum of primary triple-negative breast cancers. Nature 486: 395-399. [crossref]

36. Aparicio S, Caldas C (2013) The implications of clonal genome evolution for cancer medicine. N Engl J Med 368: 842-851.[crossref]

37. Gerlinger M, Rowan AJ, Horswell S, Larkin J, Endesfelder D, et al. (2012) Intratumor heterogeneity and branched evolution revealed by multiregion sequencing. $N$ Engl $J$ Med 366: 883-892. [crossref]

38. Marusyk A, Almendro V, Polyak K (2012) Intra-tumour heterogeneity: a looking glass for cancer? Nat Rev Cancer 12: 323-334. [crossref]

39. Dobrolecki LE, Airhart SD, Alferez DG, Aparicio S, Behbod F, et al. (2016) Patientderived xenograft (PDX) models in basic and translational breast cancer research Cancer Metastasis Rev 35: 547-573. [crossref]

40. Witkiewicz AK, Balaji U, Eslinger C, McMillan E, Conway W, et al. (2016) Integrated Patient-Derived Models Delineate Individualized Therapeutic Vulnerabilities of Pancreatic Cancer. Cell Rep 16: 2017-2031.

41. Rosfjord E, Lucas J, Li G, Gerber HP (2014) Advances in patient-derived tumorxenografts: from target identification to predicting clinical response rates in oncology. Biochem Pharmacol 91: 135-143. 
42. Chapuy B, Cheng H, Watahiki A, Ducar MD, Tan Y, et al. (2016) Diffuse large B-cell lymphoma patient-derived xenograft models capture the molecular and biological heterogeneity of the disease. Blood 127: 2203-2213. [crossref]

43. Wang D, Pham NA, Tong J, Sakashita S, Allo G, et al. (2017) Molecular heterogeneity of non-small cell lung carcinoma patient-derived xenografts closely reflect their primary tumors. Int J Cancer 140: 662-673.

44. Julien S, Merino-Trigo A, Lacroix L, Pocard M, Goere D, et al. (2012) Characterization of a large panel of patient-derived tumorxenografts representing the clinical heterogeneity of human colorectal cancer. Clin Cancer Res 18: 5314-5328. [crossref]

45. Petrillo LA, Wolf DM, Kapoun AM, Wang NJ, Barczak A, et al. (2012)Xenografts faithfully recapitulate breast cancer-specific gene expression patterns of parent primary breast tumors. Breast Cancer Res Treat 135: 913-922. [crossref]

46. Mattie M, Christensen A, Chang MS, Yeh W, Said S, et al. (2013) Molecula characterization of patient-derived human pancreatic tumorxenograft models for preclinical and translational development of cancer therapeutics. Neoplasia 15: 11381150. [crossref]

47. Eirew P, Steif A, Khattra J, Ha G, Yap D, et al. (2015) Dynamics of genomic clones in breast cancer patient xenografts at single-cell resolution. Nature 518:422-426.

48. Kim KT, Lee HW, Lee HO, Kim SC, Seo YJ, et al. (2015) Single-cell mRNA sequencing identifies subclonal heterogeneity in anti-cancer drug responses of lung adenocarcinoma cells. Genome Biol 16: 127. [crossref]

49. Nguyen LV, Cox CL, Eirew P, Knapp DJ, Pellacani D, et al. (2014) DNA barcoding reveals diverse growth kinetics of human breast tumour subclones in serially passaged xenografts. Nat Commun 5: 5871. [crossref]

50. Hanahan D, Weinberg RA (2011) Hallmarks of cancer: the next generation. Cell144: 646-674. [crossref]

51. Delitto D, Pham K, Vlada AC, Sarosi GA, Thomas RM, et al. (2015) Patient-derived xenograft models for pancreatic adenocarcinoma demonstrate retention of tumor morphology through incorporation of murine stromal elements. Am J Pathol 185: 12971303. [crossref]

52. Braekeveldt N, Wigerup C, Tadeo I, Beckman S, Sanden C, et al. (2016)Neuroblastoma patient-derived orthotopicxenografts reflect the microenvironmental hallmarks of aggressive patient tumours. Cancer Lett 375: 384-389. [crossref]

53. Sansone P, Berishaj M, Rajasekhar VK, Ceccarelli C, Chang Q, et al. (2017) Evolution of Cancer Stem-like Cells in Endocrine-Resistant Metastatic Breast Cancers Is Mediated by Stromal Microvesicles. Cancer Res77: 1927-1941. [crossref]

54. Bradford JR, Wappett M, Beran G, Logie A, Delpuech O, et al. (2016) Whole transcriptome profiling of patient-derived xenograft models as a tool to identify both tumor and stromal specific biomarkers. Oncotarget 7: 20773-20787. [crossref]

55. Calon A, Lonardo E, Berenguer-Llergo A, Espinet E, Hernando-Momblona X, et al. (2015) Stromal gene expression defines poor-prognosis subtypes in colorectal cancer. Nat Genet 47: 320-329. [crossref]

56. Isella C, Terrasi A, Bellomo SE, Petti C, Galatola G, Muratore A, Mellano A, Senetta R, Cassenti A, Sonetto C et al. (2015) Stromal contribution to the colorectal cancer transcriptome. Nat Genet47:312-319.[crossref]

57. Conway T, Wazny J, Bromage A, Tymms M, Sooraj D, et al. (2012) Xenome--a too for classifying reads from xenograft samples. Bioinformatics 28: i172-178.[crossref]

58. Bruna A, Rueda OM, Caldas C (2017)Modeling Breast Cancer Intertumor and Intratumor Heterogeneity Using Xenografts. Cold Spring HarbSymp Quant Biol. [crossref]

59. Chou J, Fitzgibbon MP, Mortales CL, Towlerton AM, Upton MP, et al. (2013) Phenotypic and transcriptional fidelity of patient-derived colon cancer xenografts in immune-deficient mice. PLoS One 8: e79874.[crossref]

60. Zembutsu H, Ohnishi Y, Tsunoda T, Furukawa Y, Katagiri T, et al. (2002) Genome-wide cDNA microarray screening to correlate gene expression profiles with sensitivity of 85 human cancer xenografts to anticancer drugs. Cancer Res62:518-527. [crossref]

61. Girotti MR, Gremel G, Lee R, Galvani E, Rothwell D, et al. (2016) Application of Sequencing, Liquid Biopsies, and Patient-Derived Xenografts for Personalized Medicine in Melanoma. Cancer Discov 6: 286-299.

62. Brown KE, Chagoya G, Kwatra SG, Yen T, Keir ST, et al. (2015) Proteomic profiling of patient-derived glioblastomaxenografts identifies a subset with activated EGFR: implications for drug development. $J$ Neurochem 133:730-738.

63. Richmond J, Robbins A, Evans K, Beck D, Kurmasheva RT, et al. (2016) Acute Sensitivity of Ph-like Acute Lymphoblastic Leukemia to the SMAC-Mimetic
Birinapant. Cancer Res 76:4579-4591. [crossref]

64. Moon HG, Oh K, Lee J, Lee M, Kim JY, et al. (2015) Prognostic and functional importance of the engraftment-associated genes in the patient-derived xenograft models of triple-negative breast cancers. Breast Cancer Res Treat 154: 13-22.

65. Gupta SK, Kizilbash SH, Carlson BL, Mladek AC, Boakye-Agyeman F, et al. (2016) Delineation of MGMT Hypermethylation as a Biomarker for Veliparib-Mediated Temozolomide-Sensitizing Therapy of Glioblastoma. J Natl Cancer Inst 108.

66. Townsend EC, Murakami MA, Christodoulou A, Christie AL, Koster J, et al. (2016) The Public Repository of Xenografts Enables Discovery and Randomized Phase II-like Trials in Mice. Cancer Cell 29: 574-586. [crossref]

67. Gao H, Korn JM, Ferretti S, Monahan JE, Wang Y, et al. (2015) High-throughput screening using patient-derived tumorxenografts to predict clinical trial drug response. Nat Med 21: 1318-1325.

68. Choi YY, Cheong JH (2017)Beyond precision surgery: Molecularly motivated precision care for gastric cancer. Eur J SurgOncol43:856-864. [crossref]

69. Rea D, Del Vecchio V, Palma G, Barbieri A, Falco M, et al. (2016) Mouse Models in Prostate Cancer Translational Research: From Xenograft to PDX. Biomed Res Int 2016:9750795. [crossref]

70. Zayed AA, Mandrekar SJ, Haluska P (2015) Molecular and clinical implementations of ovarian cancer mouse avatar models. Chin ClinOncol4: 30.[crossref]

71. Whittle JR, Lewis MT, Lindeman GJ, Visvader JE (2015) Patient-derived xenograft models of breast cancer and their predictive power. Breast Cancer Res 17: 17.[crossref]

72. Marangoni E, Poupon MF (2014) Patient-derived tumour xenografts as models for breast cancer drug development. Curr Opin Oncol 26: 556-561.[crossref]

73. Das Thakur M, Pryer NK, Singh M (2014) Mouse tumour models to guide drug development and identify resistance mechanisms. J Pathol 232: 103-111.

74. Holzapfel BM, Wagner F, Thibaudeau L, Levesque JP, Hutmacher DW (2015)Concise review: humanized models of tumor immunology in the 21 st century: convergence of cancer research and tissue engineering. Stem Cells 33: 1696-1704. [crossref]

75. Drake AC, Chen Q, Chen J (2012) Engineering humanized mice for improved hematopoietic reconstitution. Cell Mol Immunol 9: 215-224.[crossref]

76. Morton JJ, Bird G, Keysar SB, Astling DP, Lyons TR, et al. (2016)XactMice: humanizing mouse bone marrow enables microenvironment reconstitution in a patientderived xenograft model of head and neck cancer. Oncogene 35:290-300.

77. Ito R, Takahashi T, Katano I, Ito M (2012) Current advances in humanized mouse models. Cell MolImmunol9: 208-214.[crossref]

78. Shultz LD, Brehm MA, Garcia-Martinez JV, Greiner DL (2012) Humanized mice for immune system investigation: progress, promise and challenges. Nat Rev Immunol 12 786-798. [crossref]

79. Rongvaux A, Willinger T, Martinek J, Strowig T, Gearty SV, et al. (2014) Developmen and function of human innate immune cells in a humanized mouse model. Nat Biotechnol 32: 364-372.[crossref]

Copyright: (C2017 Fiore D. This is an open-access article distributed under the terms of the Creative Commons Attribution License, which permits unrestricted use, distribution, and reproduction in any medium, provided the original author and source are credited. 